\title{
Demystifying Role of Ultrasound Elastography in the Evaluation of Primary Open Angle Glaucoma
}

\author{
Khizer Razak and Meena GL* \\ Department of Radiodiagnosis, SP Medical College, India
}

*Corresponding author: GL Meena, Senior Professor \& HOD Of Department of Radiodiagnosis, Sardar Patel Medical College, Bikaner, Rajasthan, India.

Received Date: August 01, 2018

Published Date: August 28, 2018

\begin{abstract}
Objectives: The aim of this study was to compare sonoelastographic findings in the retina- choroid-sclera (RCS) complex and vitreous in glaucomatous and healthy eyes.

Methods: For this cross-sectional comparative study, 20 patients with primary open-angle glaucoma (POAG) and 20 healthy volunteers were recruited. Ultrasound elastography measurements were taken with a sonographic scanner of the RCS complex, anterior vitreous (AV), posterior vitreous (PV), retrobulbar fat tissue (RFT), optic disc, and optic nerve in each eye.

Results: The elasticity index of the RCS complex, RFT, optic disc, optic nerve, AV, and PV were similar in both groups ( $\mathrm{p}>0.05$ ), though the AV/PV strain ratio in the group of patients with glaucoma was significantly higher $(\mathrm{p}=0.04)$.

Conclusion: Glaucoma increases the $\mathrm{AV} / \mathrm{PV}$ strain ratio. In providing reproducible and consistent values, the real-time elastography technique may be helpful in elucidating the mechanisms of glaucoma in some aspects.
\end{abstract}

Keywords: Elastography; Ultrasound; Imaging; Glaucoma

Abbreviations: RCS: Retina Choroid Scleral; RFT: Retrobulbar Fat Tissue; OD: Optic Disc; AV: Anterior Vitreous; PV: Posterior Vitreous

\section{Introduction}

Glaucoma is the one of the most common cause of blindness worldwide [1,2]. Though glaucoma's pathogenesis remains poorly understood, it is known to inflict damage upon the optic nerve head $(\mathrm{ONH})$, the severity of which increases with increased intraocular pressure (IOP) $[3,4]$. Playing a central role in ONH biomechanics, the sclera-the eye's stiffest tissue-constitutes more than $70 \%$ of the outer envelope of the bulbus oculi [5]. Scleral stiffness [6] and scleral collagen fiber organization [7] correlate with IOP-induced deformation of the ONH. Scleral stiffness in particular also changes with age [8] and chronic IOP elevation [9].

Real Time Elastography (RTE) is a new technique for evaluating tissue elasticity- that is, the hardness or softness of tissue based on tissue compression [10]. Since RTE objectively measures tissue stiffness, it can be used as a diagnostic marker in clinical practice for diverse types of tissue. The RTE technique allows an absolute (quantitative) measurement (elasticity index, E) [11,12] and a relative strain ratio (E1/E2) assessment of the two neighboring tissues. Vural et al. [13] reported that the strain value of optic nerve and retrobulbar fat tissue were calculated with RTE. Also, they calculated to the strain ratio of the retrobulbar fat tissue and optic nerve of patients with glaucoma. RTE is based on the calculation of Young's elastic modulus, a physical quantity measuring stiffness [14]. There is also a strain ratio measurement, which represents the ratio of strains of the Area of Interest (ROI) to an equally measuring area in the neighboring tissue [15-17]. The elasticity index and strain ratio were used for comparison of the tissue in different patients [18]. A previous pilot study found that real-time ultrasound elastography imaging was effective in assessing the elasticity of ocular tissue [19].

Several postmortem studies have examined human scleral stiffness with inflation tests [20]. In an inflation test is done by giving 5 to $45 \mathrm{~mm} \mathrm{Hg}$ pressure to the intact posterior scleral shells while the full-field three dimensional displacements of the scleral surface were measured using laser speckle interferometry 
[20]. Primary open-angle glaucoma (POAG) is often associated with several changes in the vitreous $[21,22]$. To the best of our knowledge, however, no research has shown the elasticity of the sclera and vitreous in patients with POAG by using real-time ultrasound elastography. We thus hypothesized that the realtime ultrasound elastography technique can show changes in the elasticity of the sclera and vitreous in glaucomatous eyes, thereby illuminating the pathogenesis of POAG to some extent.

\section{Materials and Methods}

\section{Ethics}

The study was conducted in accordance with the ethical standards of the Declaration of Helsinki and approved by an institutional ethics committee. Informed written consent was obtained from all of the reviewed subjects.

\section{Study sample}

The sample of this cross-sectional comparative study consisted of 40 participants. The study group consisted of 20 participants with POAG, while the control group consisted of 20 participants with healthy eyes. One eye of each participant was selected randomly and recorded for analysis. The exclusion criteria were any history of ocular surgery, any ocular disorder other than mild cataract, or any systemic disorders. In the study group, no participant with POAG had any history of glaucomaoriented operations, including trabeculectomy, tube-shunt surgery, canaloplasty, and laser trabeculoplasty. All of these participants were currently being administered topical antiglaucoma drugs, yet were not using any systemic medications that could affect ocular measurements. All participants received an ophthalmic examination involving visual acuity assessment (Snellen Chart), autorefractometry, biomicroscopy, air-puff tonometry, Goldmann applanation tonometry, indirect retinoscopy, and pachymetry, as well as ultrasound elastography measurement.

\section{The ultrasound elastography technique}

Ultrasound elastography measurements were taken using a sonographic scanner (Logiq P5, GE Healthcare) and an 11-15 MHz linear probe, on which RTE software was installed. The linear probe which was employed in the B-scan ultrasonography is not a dedicated ocular probe. The probe is a broad-spectrum linear matrix array transducer. The frequency range is 11-15 MHZ. The length of FOV is $50 \mathrm{~mm}$. Examinations of participants were performed during a single visit and involved using RTE to measure ocular structures, including the retina-choroid-scleral (RCS) complex, retrobulbar fat tissue (RFT), optic disc (OD), (ON), and anterior and posterior vitreous (AV and PV). Sonoelastographic examinations were independently performed by a radiologist (Clinician A) who had seven years of experience and an ophthalmologist (Clinician B) who had three years of experience in RTE. Clinician A initially examined the patient with RTE. After 10 minutes, Clinician B examined the same patient. Participants were examined in the supine position. After an application of ultrasound gel, the elastography probe was placed in contact with the closed eyelid, and rhythmic, small compressions were applied manually by the operator.
Figure 1 shows the B-mode ultrasonography image and the related elastography image of the anterior vitreous versus posterior vitreous. The green bars at the top center of the elastography screen reflect the quality of measurements, as well as the effectiveness of the manually applied compressions. Five to seven green bars mean that enough compression was applied to produce the highest-quality measurements, while the colors on the RTE screen indicate the relative stiffness of the tissues. In this sense, decreased elasticity means increased stiffness. At least 10 attempted elastography examinations were made for each eye, after which one high-quality image was selected for analysis. The high-quality image was accepted as the image that included all ocular structures and did not have any artefact. Then, three successive elastographic measurements were performed using the high-quality image and the average results were recorded. To ensure standardization, the nasal part of the eye was used to evaluate the RCS complex and RFT measurements in all participants. The circumference of the RCS complex was drawn $5 \mathrm{~mm}$ from the nasal to the optic disc margin. Equal diameters of the measurement areas were selected, though some adjustments were needed for diameters due to variability in individual tissue dimensions. It was measured between distance from the skin surface of the eyelid and the central ROI in AV and the central ROI in PV. The Logiq P5 system automatically calculated the elasticity index values and strain ratio of both selected circular areas. The elasticity index (E), which can differ from $0-6$, is an absolute scale for Logiq P5.

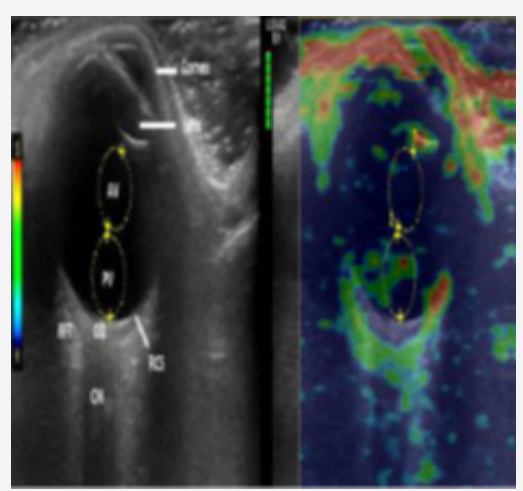

Figure 1: B-mode ultrasonography image and the related elastography image of the anterior vitreous versus posterior vitreous are demonstrated.

\section{Statistical analysis}

The Statistical Package for the Social Sciences version 17.0 for Windows (SPSS Inc., Chicago, IL, USA) was used to analyze outcomes. Any p values less than .05 were considered to be statistically significant, and all data are expressed as the mean \pm standard deviation. The Mann-Whitney U test was used to compare the ultrasound elastography measurements between the study and control groups, while the Pearson correlation test was used to demonstrate an interobserver correlation and the correlation of the $\mathrm{AV} / \mathrm{PV}$ strain ratio with IOP.

\section{Result}

The mean age of participants in the glaucoma group was $55.9 \pm 8.2$ years (range $40-69$ ) and $55.3 \pm 10.4$ years (range $38-74$ ) in 
the control group ( $p=0.79$ ). In both groups, there were eight male $(40 \%)$ and 12 female $(60 \%)$ participants $(p=1.00)$. The mean IOP values of the glaucoma and control groups were $16.1 \pm 2.1 \mathrm{mmHg}$ (range 12-20) and 14.8 $\pm 1.2 \mathrm{mmHg}$ (range 13-17), respectively (p $=0.02$ ). The mean distance $[ \pm \mathrm{SD}]$ between the central ROI in AV and skin surface of the eyelid was $13.27( \pm 1.2) \mathrm{mm}$ in glaucomatous eyes. This value was $13.12( \pm 1.1) \mathrm{mm}$ in healthy eyes.

The mean distance $[ \pm \mathrm{SD}]$ between the central ROI in PV and skin surface of the eyelid was $19.62( \pm 1.3) \mathrm{mm}$ in glaucomatous eyes and this value was $19.90( \pm 1.3) \mathrm{mm}$ in healthy eyes. There was no significant statistical difference between the central of ROI in $\mathrm{AV}$ and the skin surface of the eyelid $(\mathrm{p}=0.693)$. Also, there was no significant statistical difference between the central of ROI in PV and in the skin surface of the eyelid $(\mathrm{p}=0.489)$.

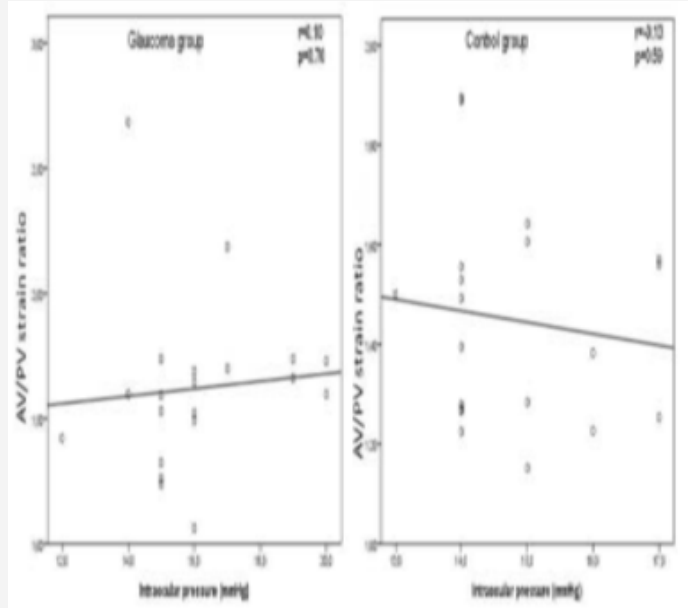

Figure 2: Scatter plot graphics of the correlation of AV/PV strain ratio with IOP are shown.

Table 1: Interobserver correlation values of elastography measurements are presented.

\begin{tabular}{|c|c|c|}
\hline Parameter & $\mathbf{r}$ & $\mathbf{P}$ \\
\hline Anterior vitreous EI & 0.70 & $<0.001$ \\
\hline Posterior vitreous EI & 0.71 & $<0.001$ \\
\hline $\begin{array}{c}\text { Retina-choroid-sclera } \\
\text { EI }\end{array}$ & 0.89 & $<0.001$ \\
\hline Retrobulbar fat EI & 0.86 & $<0.001$ \\
\hline Anterior optic disc EI & 0.81 & $<0.001$ \\
\hline Posterior optic disc EI & 0.80 & $<0.001$ \\
\hline AV/PV strain ratio & 0.60 & $<0.001$ \\
\hline
\end{tabular}

(EI=Elasticity index)

Table 2: Vitreous ultrasound elastography values of the participants are shown.

\begin{tabular}{|c|c|c|c|}
\hline Parameter & Glaucoma Group & $\begin{array}{c}\text { Control } \\
\text { Group }\end{array}$ & P \\
\hline Anterior vitreous EI & $5.19 \pm 0.24$ & $5.10 \pm 0.30$ & 0.33 \\
\hline Posterior vitreous EI & $3.32 \pm 0.63$ & $3.58 \pm 0.46$ & 0.13 \\
\hline AV / PV strain ratio & $1.62 \pm 0.34$ & $1.45 \pm 0.21$ & 0.04 \\
\hline
\end{tabular}

Interobserver correlation values of elastography measurements appear in Table 1. Interobserver correlation was the lowest among the AV/PV strain ratio, while it was the highest among the elasticity index of the RCS complex. Table 2 shows the AV and PV elasticity index of participants; though these values were similar in both groups, the AV/PV strain ratio was significantly higher in the glaucoma group. Figure 2 shows a scatter plot of the correlation of $\mathrm{AV} / \mathrm{PV}$ strain ratio with IOP. When intraocular pressure increased in the glaucoma group, the AV/PV strain ratio increased, as seen in Figure 2. But there was no significant correlation between the $\mathrm{AV} / \mathrm{PV}$ strain ratio and intraocular pressure in both the glaucoma and control groups. RCS complex and RFT elastography values of the participants appear in Table 3. There were no significant differences between glaucomatous and healthy eyes regarding RCS complex and RFT (Table 4 \&5).

Table 3: Retina-choroid-sclera complex and retrobulbar fat tissue ultrasound elastography values of the participants are demonstrated.

\begin{tabular}{|c|c|c|c|}
\hline Parameter & $\begin{array}{c}\text { Glaucoma } \\
\text { group }\end{array}$ & $\begin{array}{c}\text { Control } \\
\text { group }\end{array}$ & P \\
\hline Retina-choroid-sclera EI & $5.75 \pm 0.18$ & $5.84 \pm 0.10$ & 0.11 \\
\hline Retrobulbar fat tissue EI & $1.17 \pm 0.26$ & $1.27 \pm 0.31$ & 0.32 \\
\hline RCS / RF & $5.18 \pm 1.20$ & $4.87 \pm 1.29$ & 0.40 \\
\hline
\end{tabular}

(RCS / RFT: strain ratio of the retina-choroid-sclera complex and retrobulbar fat tissue, EI: elasticity index)

Table 4: Optic disc and nerve ultrasound elastography values of the participants are shown.

\begin{tabular}{|c|c|c|c|}
\hline Parameter & $\begin{array}{c}\text { Glaucoma } \\
\text { Group }\end{array}$ & $\begin{array}{c}\text { Control } \\
\text { Group }\end{array}$ & P \\
\hline Optic disc EI & $5.72 \pm 0.21$ & $5.79 \pm 0.20$ & 0.24 \\
\hline Optic nerve EI & $2.33 \pm 0.53$ & $2.31 \pm 0.47$ & 0.98 \\
\hline OD/ON & $2.58 \pm 0.59$ & $2.64 \pm 0.72$ & 0.94 \\
\hline
\end{tabular}

((OD / ON: strain ratio of the optic disc and optic nerve, (EI: elasticity index)))

Table 5: Age-Wise Distribution of Control \& Patients.

\begin{tabular}{|c|c|c|c|}
\hline $\begin{array}{c}\text { Age of Patients } \\
\text { According } \\
\text { to the } \\
\text { Classification } \\
\text { of the World } \\
\text { Health } \\
\text { Organization } \\
\text { (WH0) }\end{array}$ & $\begin{array}{c}\text { Control Group } \\
\text { of Healthy } \\
\text { Patients }\end{array}$ & $\begin{array}{c}\text { The Group } \\
\text { of Patients } \\
\text { with Ocular } \\
\text { Hypertension }\end{array}$ & $\begin{array}{c}\text { Group of } \\
\text { Patients with } \\
\text { Glaucoma } \\
\text { (Degree) }\end{array}$ \\
\cline { 2 - 4 } & Number of patients in the group of this age, \\
people (\%) \\
\hline $\begin{array}{c}25-44 \text { young } \\
\text { age }\end{array}$ & $4(20 \%)$ & $0(0 \%)$ & $3(15 \%)$ \\
\hline $44-60$ average \\
age
\end{tabular}

\section{Discussion}

This study chiefly showed that the elasticity of the RCS complex and vitreous may be noninvasively and safely determined using the RTE technique. Among the study's principal findings, there were, first, no differences between the elasticity of the RCS complex of glaucomatous and healthy eyes in the posterior pole. Second, OD elasticity in glaucomatous eyes was similar to that in healthy eyes. Third, the AV/ PV ratio was significantly higher in participants with POAG. 
Both scleral structure and its dynamic response to increased IOP may be important in the pathogenesis of glaucomatous damage (23-24). Using the inflation method, Zeimer and Ogura (25) found that $\mathrm{ONH}$ was stiffer in postmortem glaucomatous eyes. Tests of living human eyes by indirectly measuring the change in IOP and ocular volume suggest that ocular rigidity might be greater in eyes with POAG [26-27]. For example, Girard et al. [9] reported a positive correlation between increased chronic IOP and scleral stiffness in an animal model. Furthermore, Coudrillier et al. [28] showed by using the biomechanical inflation test that meridional strains were significantly lower in normal eyes in the peripapillary sclera. However, there was no significant difference between normal and glaucomatous eyes regarding circumferential strain. These authors also determined that glaucoma eyes were not significantly different from healthy eyes in terms of the stress-strain response in the mid posterior sclera, which may support our findings to some extent.

A reason for similar scleral elasticity between normal and glaucomatous eyes may be that patients were in the early stages of the disease and were receiving medical treatment. Another possible explanation for unchanged elasticity in the RCS complex in glaucomatous eyes may be the incapacity of RTE to measure fine ocular structure. In order to minimize this effect, very good cooperation on the part of the volunteers in their eye movements was achieved. The patients were asked to look straight ahead so that the eye was held in the primary gaze position. Secondly, optimal compression to the orbit was applied. Also, at least 10 elastographic images for each eye were taken, after which one high-quality image was selected for analysis. Three successive elastographic measurements were then performed of the high-quality image and average results were recorded. Furthermore, posterior scleral elasticity may not play an important role in the pathogenesis of POAG compared to trabecular network dysfunction.

Burgoyne et al. [29] found that acute elevations of IOP in a normal monkey eye led to small and reversible (elastic) posterior deformations of the OD surface. Our study showed no significant differences between healthy and glaucomatous eyes in the elastography of OD and ON. As such, further studies are needed to assess OD and ON elasticity in several diseases, including optic atrophy and papilledema.

Other techniques for assessing vitreous elasticity include nuclear magnetic resonance imaging for the liquefaction of the vitreous [30], radiation force [31], ultrasound image velocimetry [32], and the microbubble-based acoustic radiation force technique [33]. By using this last technique, Yoon et al. [33] reported that the $\mathrm{AV}$ has a higher share of viscosity than the PV and central vitreous. Changes in the vitreous may be observed in glaucoma. In another study, the proteolytic activity of the vitreous was found to be greater in glaucomatous eyes [34]. Measurements of AV/PV strain ratio have been used previously in the literature [19]. They reported that the posterior vitroeous was more elastic than the anterior vitroeous in patients with panretinal photocoagulation and control groups. In our study, the elastographic measurement was shown to have a relatively lower elasticity area in posterior vitreous patients with glaucoma and healthy eyes (Figure 1). Yoon et al. [33] found that the viscosity of AV and PV are different. This supports our results. However, it has been reported that depth of tissue affected the strain ratio measurements when using elastography phantom imitating liver tissue [35]. In our study, there were no significant differences between depths of AV and PV glaucomatous eyes and healthy eyes. Finally, the diversity of viscosity can affect the elastography measurement. In the present study, though glaucomatous eyes were not significantly different from healthy eyes regarding the elasticity of the $\mathrm{AV}$ and $\mathrm{PV}$, the AV/PV strain ratio was significantly higher in glaucomatous eyes. Both $\mathrm{AV}$ and PV elasticity may be minimally decreased due to glaucoma. After the use of antiglaucomatous drugs, the AV elasticity index may be minimally increased. Finally, AV/PV strain ratio may be increased. This outcome may stem from the use of antiglaucomatous drugs that mainly affect the anterior eye and thus the AV.

However, the present research should be assessed in light of several weaknesses. The RCS complex may not be affected in patients with early glaucoma, suggesting that patients at different stages of glaucoma should be examined in further studies. These studies should also include in their samples glaucomatous eyes not receiving medical treatment. The ultrasound elastography technique also poses certain limitations, including the fact that the compression applied with the probe has a relatively high operator dependency. Because the probe is not a dedicated probe for ocular imaging, the spatial resolution of the elastogram appears low. This factor may affect the elastographic measurements. In this sense, the ultrasound elastography technique needs to be improved, specifically for ocular use. Finally, real-time ultrasound elastography may not have the ability to measure absolute stiffness via Shear waving imaging.

\section{Conclusion}

The real-time ultrasound elastography technique is a safe, noninvasive procedure that can be used to analyze the mechanical properties of the RCS complex and vitreous in patients with glaucoma. The elasticity index of the RCS complex, RFT, OD, and ON was similar in both normal and glaucomatous eyes, while the AV/ PV strain ratio was higher in glaucomatous eyes.

\section{References}

1. Chan EW, Li X, Tham YC, Liao J, Wong TY, et al. (2015) Glaucoma in Asia: regional prevalence variations and future projections. Br J Ophthalmol $100(1): 78-85$.

2. Zetterberg M (2015) Age-related eye disease and gender. Maturitas 83: $19-26$

3. Mastropasqua R, Fasanella V, Agnifili L, Fresina M, Di Staso S, et al. (2015) Advance in the pathogenesis and treatment of normal-tension glaucoma. Prog Brain Res 221: 213-232.

4. Pinazo-Duran MD, Zanon-Moreno V, Gallego-Pinazo R, Garcia-Medina JJ (2015) Oxidative stress and mitochondrial failure in the pathogenesis of glaucoma neurodegeneration. Prog Brain Res 220: 127-153.

5. Norman RE, Flanagan JG, Sigal IA, Rausch SM, Tertinegg I, et al. (2011) Finite element modeling of the human sclera: influence on optic nerve head biomechanics and connections with glaucoma. Exp Eye Res 93(1): 4-12.

6. Sigal IA, Yang H, Roberts MD, Burgoyne CF, Downs JC (2011) IOP-induced lamina cribrosa displacement and scleral canal expansion: an analysis of factor interactions using parameterized eye-specific models. Invest Ophthalmol Vis Sci 52(3): 1896-1907. 
7. Grytz R, Meschke G, Jonas JB (2011) The collagen fibril architecture in the lamina cribrosa and peripapillary sclera predicted by a computational remodeling approach. Biomech Model Mechanobiol 10(3): 371-382.

8. Girard MJ, Suh JK, Bottlang M, Burgoyne CF, Downs JC (2009) Scleral biomechanics in the aging monkey eye. Invest Ophthalmol Vis Sci 50(11): 5226-5237.

9. Girard MJ, Suh JK, Bottlang M, Burgoyne CF, Downs JC (2011) Biomechanical changes in the sclera of monkey eyes exposed to chronic IOP elevations. Invest Ophthalmol Vis Sci 52(8): 5656-5669.

10. Satake H, Nishio A, Ikeda M, Ishigaki S, Shimamoto K, et al. (2011) Predictive value for malignancy of suspicious breast masses of BI-RADS categories 4 and 5 using ultrasound elastography and MR diffusionweighted imaging. AJR Am J Roentgenol 196(1): 202-209.

11. Ding J, Cheng HD, Huang J, Zhang Y, Liu J (2012) An improved quantitative measurement for thyroid cancer detection based on elastography. Eur J Radiol 81(4): 800-805.

12. Ophir J, Cespedes I, Ponnekanti H, Yazdi Y, Li X (1991) Elastography: a quantitative method for imaging the elasticity of biological tissues. Ultrason Imaging 13(2): 111-134.

13. Vural M, Acar D, Toprak U, Alp MN, Koz OG, et al. (2015) The evaluation of the retrobulbar orbital fat tissue and optic nerve with strain ratio elastography. Med Ultrason 17(1): 45-48.

14. Hall TJ (2003) AAPM/RSNA physics tutorial for residents: topics in US: beyond the basics: elasticity imaging with US. Radiographics 23(6): 1657-1671.

15. Lim DJ, Luo S, Kim MH, Ko SH, Kim Y (2012) Interobserver agreement and intraobserver reproducibility in thyroid ultrasound elastography. AJR Am J Roentgenol 198(4): 896-901.

16. Menzilcioglu MS, Duymus M, Gungor G, Citil S, Sahin T, et al. (2014) The value of real-time ultrasound elastography in chronic autoimmune thyroiditis. Br J Radiol 87(1044): 20140604.

17. Park HJ, Lee SY, Lee SM, Kim WT, Lee S, et al. (2015) Strain elastography features of epidermoid tumours in superficial soft tissue: differences from other benign soft-tissue tumours and malignant tumours. $\mathrm{Br}$ J Radiol 88(1050): 20140797

18. Kesikburun S, Yaşar E, Adıgüzel E, Güzelküçük Ü, Alaca R, et al. (2015) Assessment of Spasticity With Sonoelastography Following Stroke: A Feasibility Study. Pm R 7(12): 1254-1260.

19. Pekel G, Agladioglu K, Acer S, Yagci R, Kasikci A (2015) Evaluation of Ocular and Periocular Elasticity after Panretinal Photocoagulation: An Ultrasonic Elastography Study. Curr Eye Res 40(3): 332-337.

20. Fazio MA, Grytz R, Morris JS, Bruno L, Girkin CA, et al. (2014) Human scleral structural stiffness increases more rapidly with age in donors of African descent compared to donors of European descent. Invest Ophthalmol Vis Sci 55(11): 7189-7198.

21. Doganay S, Cankaya C, Alkan A (2012) Evaluation of corpus geniculatum laterale and vitreous fluid by magnetic resonance spectroscopy in patients with glaucoma; a preliminary study. Eye 26(8): 1044-1051.
22. Lu DW, Chang CJ, Wu JN (2001) The changes of vitreous $\mathrm{pH}$ values in an acute glaucoma rabbit model. J Ocul Pharmacol Ther 17(4): 343-350.

23. Burgoyne CF, Downs JC, Bellezza AJ, Suh JK, Hart RT. (2005) The optic nerve head as a biomechanical structure: a new paradigm for understanding the role of IOP-related stress and strain in the pathophysiology of glaucomatous optic nerve head damage. Prog Retin Eye Res 24(1): 39-73

24. Cone FE, Gelman SE, Son JL, Pease ME, Quigley HA. (2010) Differential susceptibility to experimental glaucoma among 3 mouse strains using bead and viscoelastic injection. Exp Eye Res 91(3): 415-424.

25. Zeimer RC, Ogura Y. (1989) The relation between glaucomatous damage and optic nerve head mechanical compliance. Arch Ophthalmol 107(8): 1232-1234.

26. Ebneter A, Wagels B, Zinkernagel MS. (2009) Non-invasive biometric assessment of ocular rigidity in glaucoma patients and controls. Eye 23(3): 606-611.

27. Hommer A, Fuchsjager-Mayrl G, Resch H, Vass C, Garhofer G, et al. (2008) Estimation of ocular rigidity based on measurement of pulse amplitude using pneumotonometry and fundus pulse using laser interferometry in glaucoma. Invest Ophthalmol Vis Sci 49(9): 4046-50.

28. Coudrillier B, Tian J, Alexander S, Myers KM, Quigley HA, et al. (2012) Biomechanics of the human posterior sclera: age- and glaucoma-related changes measured using inflation testing. Invest Ophthalmol Vis Sci 53(4): 1714-1728.

29. Burgoyne CF, Quigley HA, Thompson HW, Vitale S, Varma R. (1995) Measurement of optic disc compliance by digitized image analysis in the normal monkey eye. Ophthalmology 102(12): 1790-1799.

30. Aguayo J, Glaser B, Mildvan A, Cheng HM, Gonzalez RG, et al. (1985) Study of vitreous liquifaction by NMR spectroscopy and imaging. Invest Ophthalmol Vis Sci 26(5): 692-697.

31. Walker WF, Fernandez FJ, Negron LA. (2000) A method of imaging viscoelastic parameters with acoustic radiation force. Phys Med Biol 45(6): 1437-1447.

32. Rossi T, Querzoli G, Pasqualitto G, Iossa M, Placentino L, et al. (2012) Ultrasound imaging velocimetry of the human vitreous. Exp Eye Res 99: 98-104.

33. Yoon S, Aglyamov S, Karpiouk A, Emelianov S (2013)Correspondence: Spatial variations of viscoelastic properties of porcine vitreous humors. IEEE Trans Ultrason Ferroelectr Freq Control 60(11): 2453-2460.

34. Wolanska M, Bakunowicz-Lazarczyk A, Bankowski E (1991) Proteolytic activity of vitreous-humour. Acta Biochim Pol 38(1): 119-122.

35. Havre RF, Waage JR, Gilja OH, Odegaard S, Nesje LB. (2011) Real-Time Elastography: Strain Ratio Measurements Are Influenced by the Position of the Reference Area. Ultraschall Med. 